1. BDS, FCPS

Assistant Professor Operative Dentistry

Jinnah Medical and Dental College Karachi.

2. BDS, MSc

Senior Registrar Prosthodontics Jinnah Medical and Dental College, Karachi.

3. BDS, FCPS

Assistant Professor of

Prosthodontics

College of Dentistry, King Khalid

University, Abha, KSA.

4. BDS, MDS

Assistant Professor Periodontology

Armed Forces Institute of Dentistry Rawalpindi.

5. BDS, FCPS

Assistant Professor Oral Surgery Jinnah Medical \& Dental College, Karachi.

6. BDS, MSc

Senior Registrar Prosthodontics Shahida Islam Medical and Dental College, Multan.

Correspondence Address:

Dr. Farhan Butt

Department of Periodontology

Armed Forces Institute of Dentistry,

Rawalpindi.

farhanbuttpk84@gmail.com

Article received on:

05/03/2020

Accepted for publication:

$11 / 05 / 2020$

\section{TO COMPARE POST OBTURATION ENDODONTIC FLARE- UPS FOLLOWING SINGLE AND MULTIPLE VISIT ROOT CANAL TREATMENT.}

\begin{abstract}
Sajjad Ali Darvesh', Irum Naz², Abdul Razzaq Ahmed ${ }^{3}$, Farhan Butt ${ }^{4}$, Wasey Latif ${ }^{5}$, Azam Shahzad ${ }^{6}$
ABSTRACT... Objective: To compare post obturation endodontic flare-ups following single and multiple visit root canal treatment. Study Design: Quasi experimental study. Setting Operative dentistry department, DOW university hospital Karachi. Period: $2^{\text {nd }}$ October 2017 to $2^{\text {nd }}$ April 2018. Material \& Methods: A total of 100 patients of permanent exposed anterior teeth with positive pup response / vital, either gender were included. Patients divided into two groups according to single and multiple visit root canal treatment. At each post obturation recall visit the patients were interviewed and inspected to determine whether or not there are symptoms. Results: A total of 100 patients came for root canal treatment. At 1st post obturation day, out of 50 patients treated in one visit, four experienced a flare-up (8\%). Out of 50 patients treated in two visits, eight experienced a flare-up (16\%) $(P$-value $=0.12)$. At 3rd post obturation day, out of 50 patients treated in one visit, three experienced a flare-up (6\%). Out of 50 patients treated in two visits, five experienced a flare-up $(10 \%)(P$-value=0.37). Conclusion: We conclude that almost same results for single and multiple visit endodontics were achieved not statistically significant.
\end{abstract}

Key words: $\quad$ Flare-ups, Working Length, Working Width.

Article Citation: Darvesh SA, Naz I, Ahmed AR, Butt F, Latif W, Shahzad A. To compare post obturation endodontic flare-ups following single and multiple visit root canal treatment. Professional Med J 2020; 27(7):1516-1520.

DOI: 10.29309/TPMJ/2020.27.07.4618

\section{INTRODUCTION}

The ongoing fierce battle over one visit versus multiple endodontic treatments has become a very clouded issue that is debated not with scholarly precision but with business minded intent. ${ }^{1}$ In today's fast moving world, patients are requesting for treatment modalities that requiring minimum visits. ${ }^{2}$ This has created interest in clinicians to study the advisability and efficacy of root canal therapy in one visit total endodontic care of a tooth in a single visit is an old concept in clinical practice. ${ }^{3}$ Modern root canal treatment has grown rapidly over the last few years as more and more people are willing to salvage their teeth. Advances in technology, instruments and chemical composition have led to the most comprehensive and satisfying procedures for the treatment of root canals today. ${ }^{4}$

Problems associated with the root canal treatment are postoperative pain, during visiting pain and inflammation. Such cases usually do not last long, but they can embarrass the dentist and bother the patient, especially if the tooth was symptom less before the commencement of treatment. ${ }^{5}$ Postoperative pain associated with canal therapy is a bad indicator of long-term success, but the occurrence and management of pain are of interest to the health of endodontics. ${ }^{6}$

Certain factors such as preoperative pain, number of appointments use of intracranal medications and tooth localization may predispose the development of postoperative pain and flareups. Some authors reported slightly more postobturation pain following single visit than with multiple visit procedures. Others found no significant differences in the post-obturation pain experienced by patients following single or multiple visit treatment procedures. ${ }^{7}$

The natural benefit of multiple visit treatments is 
that antimicrobial coatings between appointments can reduce viral load. Based on clinical results, the use of antimicrobial coatings such as calcium hydroxide has no additional benefit. Perhaps it is not necessary to completely eradicate germs, as much as possible to reduce the bacteria and affective canal filling may be sufficient in terms of healing rather than complete eradication. ${ }^{8,9}$

There are many advantages and some disadvantages why don't more clinicians practice single appointment endodontics? It is generally thought that the pain after surgery is greater when endodontic treatment is completed in a single visit. ${ }^{10}$

This study attempts to see if any advantage exists for one visit or multiple visit treatment of vital anterior teeth as measured by rate of flareup. Age, sex and tooth type were analyzed to see their importance in causing flare-up. The same factors were also compared between one and multiple visit treatments to ascertain whether any factor caused a greater chance of flare-up.

\section{MATERIAL \& METHODS}

This study was conducted at operative dentistry department of DOW university hospital Karachi, 2nd October 2017 to 2nd April 2018.one hundred patients were selected for root canal and were randomly allocated into two groups by envelop method. Permanent exposed anterior teeth with positive pulp response/vital and both gender was included this study. Necrosed teeth, teeth with open apices, teeth with resorbed roots and teeth with periodontal disease and mobility were excluded this study. Group a patient were given single visit and group $b$ were given multiple visit treatment. A detailed medical and dental history was taken then followed by clinical examination. Gold standards of diagnosis was a presence of bleeding from the pulp chamber and the root canals determined by direct observation after access opening. Three standardized periapical radiographs pretreatment, working length determination with files in situ and post obturation were taken during the treatment. After root canal procedure cavity was access and filled with glass ionomer cement. Finally checked for any interference with articulating paper.

Patients were recalled at two closed intervals for days $1^{\text {stand }} 3^{\text {rd }}$ days. At each post obturation recall visit the patients were interviewed and inspected to determine whether or not there are symptoms like pain.

\section{RESULTS}

Out of 100 cases, $61 \%$ were female and $39 \%$ male with mean age of patients was 34.40 \pm 10.38 years. In 24 patients root canal was done in mandibular teeth while 76 had root canal in maxillary teeth. Table-l. At 1st post obturation day, out of 50 patients treated in single visit group, four experienced a flare-up (8\%). Out of 50 patients treated in multiple visits group, eight experienced a flare-up (16\%). The relationship was statistically insignificant ( $p$ value 0.2188). At 3rd post obturation day, out of 50 patients treated in one visit, three experienced a flare-up (6\%). Out of 50 patients treated in two visits, five experienced a flare-up (10\%). The relationship was statistically insignificant ( $p$ value 0.4610). Figure-1.

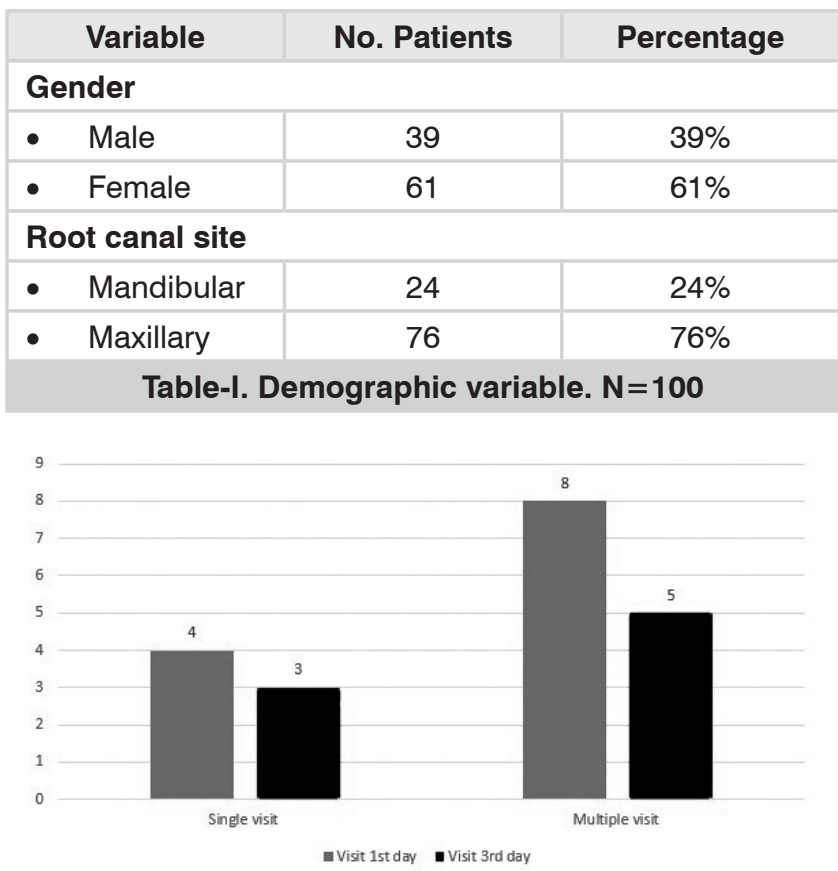

Figure-1. Comparison of flare up in both groups during follow-up.

\section{DISCUSSION}

Postoperative pain and inflammation are irritating 
to patients and clinician. Vital anterior teeth requiring root canal treatment were selected for this study. In this study post obturation endodontic flare-ups of single vs multiple visit at 1st and 3rd post obturation day was recorded through visual analogue scale. The effects of age, gender and tooth position were also analyzed.

There was no statistically significant difference observed in different age groups in this study. Our results are similar to balban, which found that patients under 50 years of age have a higher tendency to flare-up than patients over 50 years of age. ${ }^{11}$

The decrease in patient response in patients over the age of 50 is interesting. This may be associated with a decrease in pulp canal size in older patients, a decrease in blood flow in the alveoli and a decrease in the inflammatory response. $^{12}$

Eleazer and eleazer and matusow discovered no significant relationships for flare-ups with age. ${ }^{13}$ Kanefound no relationship between post obturation pain and age. Toosy ${ }^{14}$ treated necrotic teeth and made no difference except in patients of age and over 50 years old.

The reason could be a coronal transportation of the radiographic apex because of secondary cementum deposition with advancing age. This would result in an error of working length determination which could lead to extrusion of the gutt apercha obturation and sealent even if the tooth seems to be under obturated on the radiograph. ${ }^{15}$

The proponents of one visit endodontics therapy readily admit that one visit therapy is done with advantage of time saving and practice management advantages in mind many procedures which may need to be repeated for each visit are done only by once. ${ }^{16}$ Those who disagree accuse one visit therapists of advancing their business interests at the cost of ignoring scientific evidence, playing with the basic concepts and principles of endodontics and misinforming the public with catchy slogans. ${ }^{17}$
Between diehard one visit advocates and hard line one visit opponents lie those who have made unbiased attempts to discover whether one visit treatment has any advantage or disadvantage when compared to conventional multiple visit therapy, which cases justify one visit treatment and if any extra precautions need to be taken like prophylactic antibioticsto make one visit therapy more successful. ${ }^{18}$

The criteria for selecting cases for one visit therapy are as varied as the number of authors who support or oppose it. These range from a total rejection of one visit therapyto doing every case in one visit regardless of the preoperative symptoms. ${ }^{19}$ Most authors, however, agree that as long as a skilled practitioner with the necessary endodontics know-how is doing the treatment, it may be done for all cases except for those teeth in which an uncontrolled exudates or bleeding is present. In addition care must be taken that enough time is at the disposal of the endodontist to complete the procedure calmly and no contraindicating condition is presenting the patient to keep the mouth open for a long enough time to complete the procedure. ${ }^{20}$

In this study the maxillary teeth have more flare-up at 1 st and 3rd post obturation day as compared to mandibular teeth. It is not statistically significant. These results are on the contrary to the finding of walton, barnett, fox, mollar and toosyand the reason for more pain mandibular teeth than maxillary may be cortical thick plate in mandible than in maxilla. ${ }^{21}$ This may cause accumulation of exudates, which causes more pressure as compared to maxilla. Kanenfound no correlation of post obturation pain with tooth type.

Many studies have been carried out to ascertain the success rate of one visit treatment alone or in comparison with multiple visit endodontics. Most found no advantage for one visit or multiple visit regarding flare-ups. ${ }^{22}$ This study also found that there was no advantage or disadvantage offered by one visit or multiple visit treatment regarding flare-ups. Eleazer \& eleazer who reported fewer flare-ups for single visit groups 3.0\% and $8.0 \%$ for the multiple visit group. Other studies also have 
reported lower incidence of figures for endodontic flare-up walton \& fouad in the united states of America reported an incidence of $3.17 \% .{ }^{23}$

Soltanoff, however found a $64 \%$ pain incidence after one visit treatment and a $38 \%$ pain incidence after two visit treatment. These figures seems high when compared with other studies. One possible reason for this is the use of normal saline instead of sodium hypochlorite. ${ }^{24}$ The lack of an antibacterial irrigant may have contributed to a higher incidence of post-operative pain. Di renzo et al have reported that there was no statistical difference of post-operative pain between one and two visit root canal therapy. ${ }^{25}$ Oliet compared one visit to multi visit procedures for root canal treatment he found that when pain occurred post operatively, it typically presented within the first $24 \mathrm{hrs}$ and there was no difference between one visit and multi visit approach. ${ }^{26}$ Pekruhn compared one visit and multiple visit treatment and he found that both have $16 \%$ population with pain at first day. Fear of dentist, dental procedures, anxiety, apprehension and many other psychological factors influence the patient's pain perception and reaction threshold. ${ }^{27}$ Reassurance is the critical, perhaps the most important aspect of treatment. The dentist must explain that flare-ups do occur and are treatable. To reduce flare-ups included pre warning the patient about the amount of time left for effectiveness of the local anesthetic, possible post-operative pain instructions to take a non steroidalanti inflammatory drug if required. Oney carried out a study if certain measures contributed to the reduction of flare-up rate. He used a one visit protocol for each patient and found $0 \%$ flare-up rate. ${ }^{28}$ According to them many years of clinical experience is necessary for such endodontic competence to develop.

\section{CONCLUSION}

Flare-up has no significant influence on the outcome of endodontic treatment but its occurrence is extremely undesirable for both the patient and the clinician and can undermine clinician patient relationships. The present study reported similar results for single and multiple visit endodontics and the results are not statistically significant. No advantage of age group, gender and tooth type was present. There was no correlation found between flare-up and age, gender and tooth type.

Copyright@ 11 May, 2020.

\section{REFERENCES}

1. Moreira $\mathrm{ms}$, anuar as, tedesco tk, dos santos $\mathrm{m}$, morimoto s. Endodontic treatment in single and multiple visits: an overview of systematic reviews. J endodontics. 2017 Jun 1; 43(6):864-70.

2. Wong yj. Similar treatment outcomes when performing endodontic therapy on permanent teeth in a single visit or multiple visits. $\mathrm{J}$ am dent asso. 2017; 148(9):687-9.

3. Harlamb sc. Management of incompletely developed teeth requiring root canal treatment. Australian dental j. 2016 mar; 61:95-106.

4. Sathorn c, parashos p. Monitoring the outcomes of root canal re $\square$ treatments. Endodontic topics. 2008 sep; 19(1):153-62.

5. Jayakodi $h$, kailasam s, kumaravadivel $k$, thangavelu b, mathew s. Clinical and pharmacological management of endodontic flare-up. J pharmacy \& bioallied scie. 2012; 4(suppl 2):s294.

6. Wong aw, zhang s, li sk, zhu x, zhang c, chu ch. Incidence of post-obturation pain after singlevisit versus multiple-visit non-surgical endodontic treatments. Bmc oral health. 2015 Dec; 15(1):96-9.

7. Jain $p$, mulay s, shetty $r$. Incidence of post-operative pain after single visit root canal treatment using rotary and reciprocating single file systems-a systematic review. J advanced med and dent scie resea. 2018 Jan; 6(1):25-9.

8. Dorn so, Cheung gs. Management of endodontic emergencies. Management. 2016; 8(30):48-91.

9. Neelakantan $\mathrm{p}$, romero $\mathrm{m}$, vera j, daood $\mathrm{u}$, khan $\mathrm{au}$, yan a, cheung gs. Biofilms in endodontics-current status and future directions. Intj mol scien. 2017; 18(8):1748-52.

10. Madarati aa, zafar ms, sammani am, mandorah ao, bani-younes ha. Preference and usage of intracanal medications during endodontic treatment. Saudi medj. 2017 jul; 38(7):755-9.

11. Qasim M, Anjum O, Yousaf O, Khan S, Pirvani M. Comparing the effect of Placebo and Prophylactic Antibiotics on asymptomatic Necrotic teeth. The Professional Medical Journal. 2019 Dec 10;26(12):21628. 
12. Yu cy, abbott pv. Responses of the pulp, periradicular and soft tissues following trauma to the permanent teeth. Australian dent j. 2016 mar; 61:39-58.

13. Munawwar $m$, munir mb, sajid $m$. Comparison of stepdown versus step-back hand preparation technique of root canals. Annals Abbasi Shaheed Hosp \& Karachi med \& dent coll. 2019 mar 31; 24(1):45-50.

14. Torabinejad $\mathrm{m}$, kettering jd, mcgraw jc, cummings $\mathrm{rr}$, dwyer tg, tobias ts. Factors associated with endodontic interappointment emergencies of teeth with necrotic pulps. J endod 1988: 14: 261-266.

15. Alothmani os, chandler $\mathrm{np}$, friedlander It. The anatomy of the root apex: A review and clinical considerations in endodontics. Saudi endodontic j. 2013; 3(1):1-5.

16. Londhe $s m$, sanjay $m$, sharma $s$, lal $s$. Single-visit versus dual-visit endodontics-a comparative study. Ind j pub health resea \& development. 2019; 10(7):5-9.

17. Wong aw, zhang $\mathrm{c}$, chu ch. A systematic review of nonsurgical single-visit versus multiple-visit endodontic treatment. Clin cosmetic \& invesational dentistry. 2014; 6:45.

18. Goldfein j, speirs c, finkelman m, amato r. Rubber dam use during post placement influences the success of root canal-treated teeth. J endod. 2013; 39(12):14814.

19. Wong yj. Similar treatment outcomes when performing endodontic therapy on permanent teeth in a single visit or multiple visits. $\mathrm{J}$ am dent asso. 2017 sep $1 ;$ 148(9):687-9.

20. Ingle ji, beveridge ee, glick dh, weichman ja, abou-rass m. Modern endodontic therapy. J endodontics. 1994; 2:1-57.
21. Khan m, akbar i, rehman k. Effect of prophylactic amoxicillin on endodontic flare-up in teeth with asymptomatic, necrotic pulps and periradicularpathosis. Pak oral \& dent j. 2010 Dec 1; 30(2):285-290.

22. Schwendicke f, göstemeyer g. Single-visit or multiplevisit root canal treatment: Systematic review, metaanalysis and trial sequential analysis. Bmj open. 2017 Feb 1; 7(2):e013115.

23. Walton $r$, fouad a: endodontic interappointment flareups: A prospective study of incidence and related factors. J endod 1992, 18:172-7.

24. Alharthi aa, aljoudi $\mathrm{mh}$, almaliki $\mathrm{mn}$, almalki ma, sunbul ma. Effect of intra-canal cryotherapy on postendodontic pain in single-visit RCT: A randomized controlled trial. Saudi dent j. 2019 Jul 1; 31(3):330-5.

25. Direnzo a, gresla $t$, johnson br, rogers $m$, tucker $d$, begole ea. Postoperative pain after 1-and 2-visit root canal therapy. Oral surg oral med oral pathol, oral radiol \& endodontol. 2002 may 1; 93(5):605-10.

26. Patil aa, joshi sb, bhagwat sv, patil sa. Incidence of postoperative pain after single visit and two visit root canal therapy: A randomized controlled trial. Jclin and diagresea: jcdr. 2016 may; 10(5):zc09.

27. Appukuttan dp. Strategies to manage patients with dental anxiety and dental phobia: Literature review. Clin, cosmetic \& investigational dentistry. 2016; 8:35.

28. Onay eo, ungor $m$, yazici ac. The evaluation of endodontic flare-ups and their relationship to various risk factors. Bmc oral health. 2015 dec 1; 15(1):142-5.

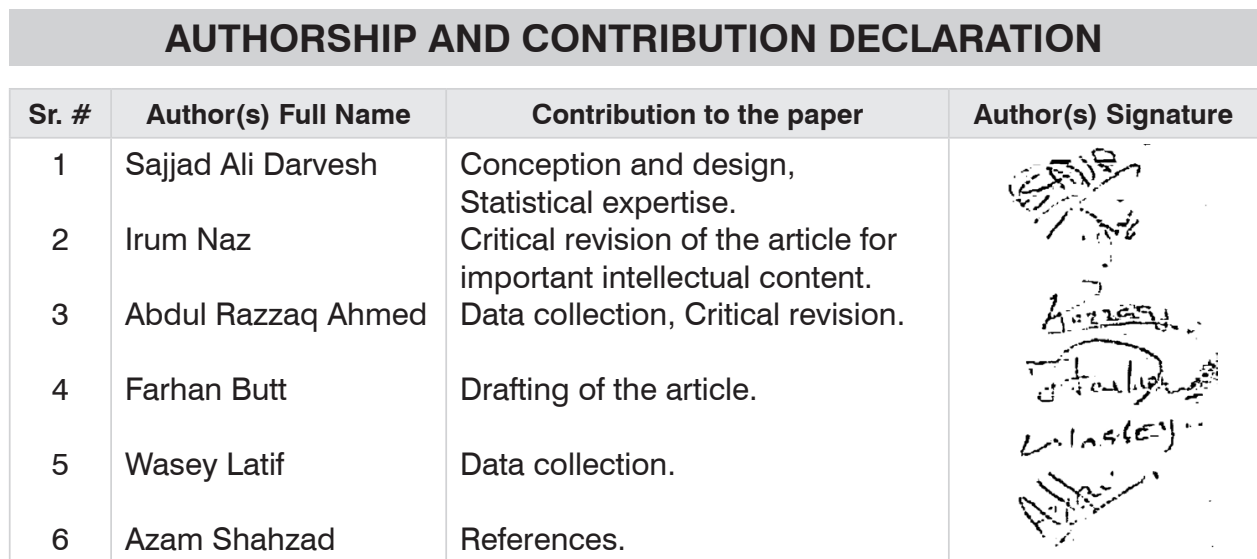

